\title{
STABILITY ISSUES OF FINITE PRECISION CONTROLLER STRUCTURES USING THE DELTA OPERATOR FOR SAMPLED DATA SYSTEMS
}

\author{
Jun Wu*, Robert Habib Istepanian**, Jian Chu*, \\ James F. Whidborne ${ }^{* *}$, Sheng Chen ${ }^{* * * *}$, Jianbo $\mathrm{Hu}^{*}$

\begin{abstract}
This paper present a new effective algorithm for the optimal realization of sampled-data SISO two order controller structures using the delta operator subject to Finite-Word-Length (FWL) constraints. The problem is formulated as a non-linear programming problem with a maximum of four independent variables to provide an easy and efficient optimization tool to solve such complex problems. Simulation results of the optimum FWL realizations of digital SISO two order controller structure applied to an electrohydraulic actuator system are presented to illustrate the effectiveness of the proposed strategy. Copyright $@ 1999$ IFAC
\end{abstract}

Keywords: control applications of computers, stability robustness, sampled data systems, optimization problems, linear systems.

\section{INTRODUCTION}

The recent advances in fixed-point implementation of digital controllers such as the design of dedicated fixcd-point Digital Signal Processors (DSP) and new Digital Control Processors (DCP) architectures made Finite Word Length (FWL) implementation an important issue in modem digital control engineering design applications. Improved control performance and increased levels of integration are especially important in many areas such as consumer electronic products, automotive and electro-mechanical control systems. This is because hardware controller implementation with fixed-point arithmetic offer the advantages of spced. memory space, cost and simplicity over floating-point arithmetic.

The FWL effects have been well studied in digital 
signal processing, especially in digital filter implementation since the 1970's (Roberts and Mullis, 1987). The results have recently been extended to the study of FWL effects of digital controller on control systems. Moroney et al. (1980) studied the effects of FWL implemented digital controller on the degradation of an LQG cost function from a statistical point of view. Fialho and Georgiou (1994) analyzed the effects of FWL implemented digital controller on the stability and performance of sampled data systems. A FWL stability measure $\mu_{0}$ was presented by Moroncy et al. (1980) and Fialho and Georgiou (1994), but computing explicity this measure seems very hard and is still an open problem. Based on the first order approximation, $\mathrm{Li}$ and Gevers (1996) and Istepanian et al. (1998b) developed two tractable FWL stability measures which are the lower bounds of $\mu_{0}$ respectively. More recently, Istepanian et al. (1996, 1998a, 1998c), Madievski et al. (1995), Qiu et al. (1993), and Whidborne et al. (1998) dealt with FWL implementation issues using different approaches.

In all these studies of FWL effects of digital controllers were described and realized using the usual shift operator $z$ structure. It is well known that a discrete time system can also be described and realized with a different discrete operator called delta operator (Middleton and Goodwin, 1990). The delta operator parametrization provides a unified formulation between the continuous time and discrete time systems. This paper presents the FWL stability issues of sampling data control systems using the delta operator domain. The results of $\mathrm{Li}$ and Gevers (1996) and Istepanian et al. (1998b) are extended using the delta operator formulation and a deltabased FWL stability measure is derived. The relevant procedures for computing this measure and the algorithmic approach for the optimal FWL realizations providing the maximum stability measures are also presented. The theoretical approach is illustrated by a numerical example.

\section{THE DELTA OPERATOR FORMULATIONS}

From a continuous time transfer function $G(s)$, as the results of a discretization proccdure with the shift operator $z$ and a sampling period $h$, a discrete time transfer function $G_{z}(z)$ can be obtained. Dcfinc

$$
\delta=\frac{z-1}{h}
$$

with this definition, the transfer function $G_{z}(z)$ can be re-expressed in $\delta$ form:

$$
G_{z}(z)=G_{\delta}(\delta)
$$

which means $G_{z}(z)$ and $G_{\mathcal{S}}(\delta)$ are two different but equivalent parametrizations representing the same object. These two input-output relationships can be represented by a shift operator (resp. $\delta$ operator) state space model as follows:

$$
\begin{aligned}
& \left\{\begin{array}{l}
z x_{z}(k)=A_{z} x_{z}(k)+B_{z} u(k) \\
y(k)=C_{z} x_{z}(k)+D_{z} u(k)
\end{array}\right. \\
& \left\{\begin{array}{l}
\delta x_{\delta}(k)=A_{\delta} x_{\delta}(k)+B_{\delta} u(k) \\
y(k)=C_{\delta} x_{\delta}(k)+D_{\delta} u(k)
\end{array}\right.
\end{aligned}
$$

The following relationships relate the internal and external representations:

$$
\begin{aligned}
G_{z}(z) & =C_{z}\left(z I-A_{z}\right)^{-1} B_{z}+D_{z} \\
& =G_{\delta}(\delta)=C_{\delta}\left(\delta I-A_{\delta}\right)^{-1} B_{\delta}+D_{\delta} \\
A_{z} & =h A_{\delta}+I \\
B_{z} & =h B_{\delta} \\
C_{z} & =C_{\delta} \\
D_{z} & =D_{\delta}
\end{aligned}
$$

Define

$$
\begin{aligned}
S_{\rho}=\{ & \left(A_{\rho}, B_{\rho}, C_{\rho}, D_{\rho}\right): \\
& \left.G_{\rho}(\rho)=C_{\rho}\left(\rho I-A_{\rho}\right)^{-1} B_{\rho}+D_{\rho}\right\}
\end{aligned}
$$

where $\rho=z$ or $\delta$ is called generalized operator. Hence if $\quad\left(A_{\rho}, B_{\rho}, C_{\rho}, D_{\rho}\right) \in S_{\rho}$, $\left(T^{-1} A_{\rho} T, T^{-1} B_{\rho}, C_{\rho} T . D_{\rho}\right) \in S_{\rho}$ if and only if $T$ is nonsingular. The following lemmas result directly.

Lemma 1: $\lambda_{i}\left(A_{z}\right)=1+h \lambda_{i}\left(\Lambda_{\delta}\right)$, where $\lambda_{i}(\cdot)$ is the eigenvalue.

It is well known that the discrete time system $\left(A_{z}, B_{z}, C_{z}, D_{z}\right)$ is stable if and only if $\left|\lambda_{i}\left(A_{z}\right)\right|<1$. From lemma 1, we can get the condition of the stability of the discrete time system described with the delta operator:

$$
\begin{aligned}
& \text { Lemma 2: The discrete time system } \\
& \left(A_{\mathcal{S}}, B_{\delta}, C_{\mathcal{S}}, D_{\mathcal{S}}\right) \text { is stable if and only if } \\
& \left|\lambda_{i}\left(A_{\delta}\right)+\frac{1}{h}\right|<\frac{1}{h} \text {. }
\end{aligned}
$$

\section{DELTA-BASED STABILITY ROBUSTNESS MEASURES WITH FWL CONSIDERATION}

Considering the sampled data system $\Sigma_{1}$ shown as Figure 1.

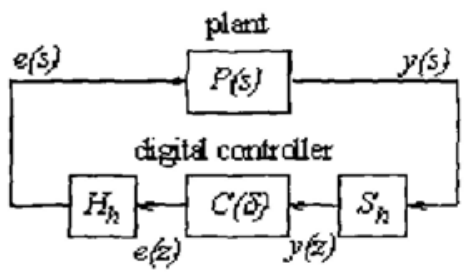

Fig. 1. Sampled data system 
where $P(s)$ is the continuous time plant, $C(\delta)$ is the discrete time controller, $S_{h}$ is the sampler with sampling period $h$,

$$
y(z)=S_{h} y(s): y(k)=y(k h)
$$

$H_{h}$ is the hold device with sampling period $h$.

$$
e(s)=H_{h} e(z): e(t)=e(k),(k h<t \leq(k+1) h)
$$

Suppose $P(s)$ is strictly proper. Let $\left(A_{P}, B_{P}, C_{P}, 0\right)$ be a state description of $P(s)$ i.e. $P(s)=C_{P}\left(s I-A_{P}\right)^{\prime} B_{P} \quad$ where $A_{P} \in R^{m \times m}, B_{P} \in R^{m \times l}, C_{P} \in R^{q \times m}$ Let $\left(A_{C}, B_{C}, C_{C}, D_{C}\right)$ be a state description of $C(\delta)$, i.e. $C(\delta)=C_{C}\left(\delta I-A_{C}\right)^{-1} B_{C}+D_{C} \quad$ where $A_{C} \in R^{n \times n}, B_{C} \in R^{n \times q}, C_{C} \in R^{l \times n}, D_{C} \in R^{i \times q}$. In this paper, $\left(A_{C}, B_{C}, C_{C}, D_{C}\right)$ is also called a realization of $C(\delta)$. The realizations of $C(\delta)$ are not unique. In fact, if $\left(\Lambda_{C}^{0}, B_{C}^{0}, C_{C}^{0}, D_{C}^{0}\right)$ is a realization of $C(\delta)$. Then so is $\left(T^{-1} A_{C}^{0} T, T^{-1} B_{C}^{0}, C_{C}^{0} T, D_{C}^{0}\right)$ for any similarity transformation $T \in R^{n \times n}$. Considering the behaviour of the sampled data system $\Sigma_{1}$ only at its sampling instants, we arrive at a discrete time fecdback system $\Sigma_{2}$ :

$$
\left\{\begin{array}{l}
y(z)=S_{h} P(s) H_{h} e(z) \\
e(z)=C(\delta) y(z)
\end{array}\right.
$$

The plant $P(\delta)=S_{h} P(s) I_{h}=C_{\delta}\left(\delta I-A_{\delta}\right)^{-1} B_{\delta}$ is the discretized $P(s)$ whose state description is $\left(A_{s}, B_{\mathcal{S}}, C_{\mathcal{S}}, 0\right)$, where

$$
\begin{aligned}
& A_{\delta}=\frac{1}{h}\left(e^{A_{p} h}-I\right) \in R^{m \times m}, \\
& B_{\delta}=\frac{1}{h} \int_{0}^{h} e^{A_{p} z} B_{P} d \tau \in R^{m \times l}, C_{\delta}=C_{P} \in R^{q \times m}
\end{aligned}
$$

It can be easily seen that the corresponding state description $(\bar{A}, \bar{B}, \bar{C}, \bar{D})$ of the discrete time closed loop system $\Sigma_{2}$ without FWL effect is given by:

$$
\begin{aligned}
\bar{A} & =\left[\begin{array}{cc}
A_{\delta}+B_{\delta} D_{C} C_{\delta} & B_{\delta} C_{C} \\
B_{C} C_{\delta} & A_{C}
\end{array}\right] \\
& =\left[\begin{array}{cc}
A_{\delta} & 0 \\
0 & 0
\end{array}\right]+\left[\begin{array}{cc}
B_{\delta} & 0 \\
0 & I_{n}
\end{array}\right]\left[\begin{array}{ll}
D_{C} & C_{C} \\
B_{C} & A_{C}
\end{array}\right]\left[\begin{array}{cc}
C_{\delta} & 0 \\
0 & I_{n}
\end{array}\right] \\
& =M_{0}+M_{1} X M_{2}=\bar{A}(X) \\
& \bar{B}=\left[\begin{array}{c}
B_{\delta} \\
0
\end{array}\right] \bar{C}=\left[\begin{array}{ll}
C_{\delta} & 0
\end{array}\right] \bar{D}=0
\end{aligned}
$$

where $\quad M_{0} \in R^{(m+n) \times(m+n)}, M_{1} \in R^{(m+n) \times(i+n)}$, $M_{2} \in R^{(q+n) \times(m+n)}$ are fixed matrices that depcnd on $P(s)$ and $h ; X \in R^{(l+n) \times(q+n)}$ is called the controller matrix: $I_{n}$ denotes $n \times n$ identity matrix. Suppose $C(\delta)$ has been given to make the sampled data system $\Sigma_{1}$ stable, and the realization of $C(\delta)$ is
$X_{0}=\left[\begin{array}{ll}D_{C}^{0} & C_{C}^{0} \\ B_{C}^{0} & A_{C}^{0}\end{array}\right]$. Since the sampled data system $\Sigma_{1}$ is stable if and only if system $\Sigma_{2}$ is stable (Chen and Francis, 1991), it follows from lemma 2 that $\forall i \in\{1, \cdots, m+n\}, \quad\left|\lambda_{i}\left(\bar{A}\left(X_{0}\right)\right)+\frac{1}{h}\right|<\frac{1}{h}$. When realization $\left(A_{C}^{0}, B_{C}^{0}, C_{\bigodot}^{0}, D_{C}^{0}\right) \quad$ of $C(\delta)$ is implemented with a DCP, the controller matrix

$$
X=\left[\begin{array}{cccc}
p_{1} & p_{2} & \cdots & p_{q+n} \\
p_{q+n+1} & p_{q+n+2} & \cdots & p_{2(q+n)} \\
\vdots & \vdots & \cdots & \vdots \\
p_{(l+n-1)(q+n)+1} & p_{(l+n-1)(q+n)+2} & \cdots & p_{(l+n)(q+n)}
\end{array}\right]
$$

is perturbed into:

$$
\begin{aligned}
& X_{0}+\Delta X=\left[\begin{array}{cccc}
D_{C}^{0} & \left.C_{C}^{0}\right]+ \\
B_{C}^{0} & A_{C}^{0} J
\end{array}\right. \\
& {\left[\begin{array}{cccc}
\Delta p_{1} & \Delta p_{2} & \cdots & \Delta p_{q+n} \\
\Delta p_{q+n+1} & \Delta p_{q+n+2} & \cdots & \Delta p_{2(q+n)} \\
\vdots & \vdots & \cdots & \vdots \\
\Delta p_{(l+n-1)(q+n)+1} & \Delta p_{(l+n-l)(q+n)+2} & \cdots & \Delta p_{(l-n)(q+n)}
\end{array}\right]}
\end{aligned}
$$

due to the FWL effect, where each element of $\Delta X$ is bounded by $\frac{\varepsilon}{2}$, i.e.

$$
\mu(\Delta X) \stackrel{\Delta}{=} \max _{i=\{1, \cdots,(I+n)(q+n)\}}\left|\Delta p_{i}\right| \leq \frac{\varepsilon}{2}
$$

For a fixed point processor of $B_{s}$ bits,

$$
\varepsilon=2^{-\left(B_{s}-B_{x}\right)}
$$

where $2^{k_{x}}$ is a normalization factor such that each parameter of $2^{-B_{X}} X_{0}$ is absolutely not bigger than 1. With the perturbation $\Delta X, \lambda_{i}\left(\bar{A}\left(X_{0}\right)\right)$ may be moved to $\lambda_{i}\left(\bar{A}\left(X_{0}+\Delta X\right)\right)$. The sampled data system $\Sigma_{1}$ is unstable if and only if there is $i \in\{1, \cdots, m+n\}$ such that $\left|\lambda_{i}\left(\bar{A}\left(X_{0}+\Delta X\right)\right)+\frac{1}{h}\right| \geq \frac{1}{h}$.

When $\Delta X$ is small, $\forall i \in\{1, \cdots, m+n\}$, we have (Li and Gevers, 1996 and Istepanian et al., 1998a)

$$
\begin{aligned}
\Delta \lambda_{i} & =\lambda_{i}\left(\bar{A}\left(x_{0}+\Delta X\right)\right)-\lambda_{i}\left(\bar{A}\left(X_{0}\right)\right) \\
& =\left.\sum_{j=1}^{N} \frac{\partial \lambda_{i}}{\partial p_{j}}\right|_{X=X_{0}} \Delta p_{j}
\end{aligned}
$$

where $N=(l+n) \times(q+n)$ is the number of elements of $X$. It follows that:

$$
\begin{aligned}
\left|\Delta \lambda_{i}\right| & \leq \sum_{j=1}^{N}\left|\frac{\partial \lambda_{i}}{\partial p_{j}}\right|_{X=X_{0}}\left|\Delta p_{j}\right| \\
& \leq \mu(\Delta X) \sum_{j=1}^{N}\left|\frac{\partial \lambda_{i}}{\partial p_{j}}\right|_{X=X_{0}} \mid
\end{aligned}
$$


Thus $\forall i \in\{1, \cdots, m+n\}$, if

$$
\mu(\Delta X)<\frac{\frac{1}{h}-\left|\lambda_{i}\left(\bar{A}\left(X_{0}\right)\right)+\frac{1}{h}\right|}{\sum_{j=1}^{N}\left|\frac{\partial \lambda_{i}}{\partial \partial_{j}}\right|_{X=X_{0}} \mid}
$$

we have

$$
\left|\lambda_{4}\left(\vec{A}\left(X_{0}+\Delta X\right)\right)+\frac{1}{h}\right| \leq \frac{1}{h}
$$

which means the sampled data system $\Sigma_{t}$ is stable. Let

$$
\mu_{1}\left(X_{0}\right)=\min _{i \in\{1, \cdots, m+n\}} \frac{\frac{1}{h}-\left|\lambda_{i}\left(\bar{A}\left(X_{0}\right)\right)+\frac{1}{h}\right|}{\sum_{j=1}^{N}\left|\frac{\partial \lambda_{i}}{\partial p_{j}}\right|_{X=X_{0}} \mid}
$$

From (16), one can reach to the following:

Theorem 1: System $\Sigma_{1}$ with FWL effect is stable when $\mu(\Delta X)<\mu_{1}\left(X_{0}\right)$.

One can compute $\mu_{1}\left(X_{0}\right)$ using the following lemma. which was proved by $\mathrm{Li}$ and Gevers (1996) and Istepanian et al. (1998a).

Lemma 3: Assume $\bar{A}(X)$ described in (7) be diagonalizable and have $\left\{\lambda_{i}\right\}=\left\{\lambda_{i}(\bar{A}(X))\right.$ as its eigenvalues, let $x_{i}$ be a right eigenvector of $\bar{A}(X)$ corresponding to the eigenvalue $\lambda_{1}$. Denote $M_{x}=\left[\begin{array}{llll}x_{1} & x_{2} & \cdots & x_{m-n}\end{array}\right] \quad$ and $M_{y}=\left[\begin{array}{llll}y_{1} & y_{2} & \cdots & y_{m+n}\end{array}\right]=M_{x}^{-H}$, where $y_{i}$ is called the reciprocal left eigenvector corresponding to $\lambda_{i}$. Then $\forall i \in\{1, \cdots, m+n\}$

$$
\frac{\partial \lambda_{i}}{\partial X}=M_{1}^{T} y_{i} x_{i}^{T} M_{2}^{T}
$$

where $y_{i}^{\prime}$ is conjugate to $y_{i}$, "T" denotes the transpose operation.

Based on $\mu_{1}\left(X_{0}\right)$, one can compute $\hat{B}_{s 1}^{\min }$, the smallest integer which is not less than $-\log _{2} \mu_{1}\left(X_{0}\right)-1+B_{X}$. From (11), (12) and theorem 1, we know that the sampled data system $\Sigma_{1}$ is stable when $X_{0}$ is implemented with a DCP of at least $\hat{B}_{s l}^{\min }$ bits.

\section{OPTIMAL DELTA REALIZATION OF CONTROLLER WITH FWL CONSIDERATION}

In this section we will discuss the optimal realization problem of digital SISO (Single Input Single Output) two order controllers because of the wider industrial use and simple application of such controllers. The usual PID controller is a kind of two order controller.

As a SISO two order controller, the initial realization $A_{C}^{0} \in R^{2 \times 2}, B_{C}^{0} \in R^{2 \times 1} . C_{C}^{0} \in R^{1 \times 2}, D_{C}^{0} \in R$. Then $X_{0}=\left[\begin{array}{cc}D_{C}^{0} & C_{C}^{0} \\ B_{C}^{0} & A_{C}^{0}\end{array}\right] \in R^{3 \times 3}$ and any realization of $C(\delta)$ is $X_{T} \stackrel{\Delta}{=}\left[\begin{array}{cc}\mathrm{I} & 0 \\ 0 & T^{-1}\end{array}\right] X_{0}\left[\begin{array}{ll}\mathrm{I} & 0 \\ 0 & T\end{array}\right]$ where $T \in R^{2 \times 2}$ and $\operatorname{det}\left(T^{\prime}\right) \neq 0$. Then from $(7)$,

$$
\bar{A}\left(X_{T}\right)=\left[\begin{array}{cc}
I_{m} & 0 \\
0 & T^{-1}
\end{array}\right] \bar{A}\left(X_{0}\right)\left[\begin{array}{cc}
I_{m} & 0 \\
0 & T
\end{array}\right]
$$

Let $x_{i}^{0}$ be a right eigenvector of $\bar{A}\left(X_{0}\right)$ corresponding to the eigenvalue $\lambda_{i}^{0}=\lambda_{i}\left(\bar{A}\left(X_{0}\right)\right)$, $y_{i}^{0}$ be the reciprocal left eigenvector corresponding to $x_{i}^{0}$. Applying lemma 3 , we have

$$
\begin{aligned}
& \left.\frac{\partial \lambda_{Y}}{\partial X}\right|_{X=X_{T}} \\
= & {\left.\left.\left[\begin{array}{cc}
E_{S}^{T} & 0 \\
0 & I_{2}
\end{array}\right] \begin{array}{cc}
I_{m} & 0 \\
0 & T^{T}
\end{array}\right]\left(y_{i}^{0}\right)^{0}\left(x_{i}^{0}\right)^{T}\left[\begin{array}{cc}
I_{m} & 0 \\
0 & T^{T}
\end{array}\right] \begin{array}{cc}
C_{S}^{T} & 0 \\
0 & I_{2}
\end{array}\right] } \\
= & {\left.\left[\begin{array}{cc}
1 & 0 \\
0 & T^{T}
\end{array}\right] \frac{\partial \lambda_{2}}{\partial X}\right|_{X=X_{0}} \quad\left[\begin{array}{cc}
1 & 0 \\
0 & T^{T}
\end{array}\right] }
\end{aligned}
$$

For complex matrix

$$
M=\left[\begin{array}{ccc}
M_{11} & \cdots & M_{1 n} \\
\vdots & \cdots & \vdots \\
M_{m 1} & \cdots & M_{m n}
\end{array}\right] \in C^{m n}
$$

denote

$$
\|M\|_{s}=\sum_{i=1}^{m} \sum_{j=1}^{n}\left|M_{i j}\right|
$$

From (17), (18), (20) and (22), we can describe the optimal FWL realization problem of digital SISO two order controller as an optimization problem.

$$
\begin{aligned}
& v=\frac{1}{\max _{X_{T}} \mu_{1}\left(X_{T}\right)}
\end{aligned}
$$

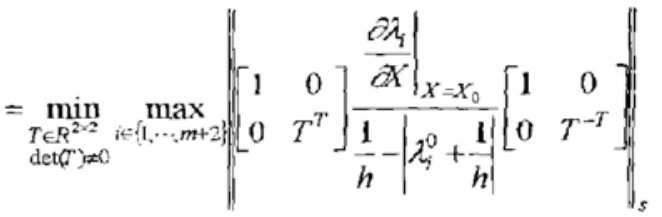

$$
\begin{aligned}
& \stackrel{\wedge}{=} \min _{\substack{T \in R^{2 \times 2} \\
\operatorname{det}(T), 00}} \max _{i \in\{1 \cdots, \cdots+2\}}\left\|\left[\begin{array}{cc}
1 & 0 \\
0 & T^{T}
\end{array}\right] \Phi_{i}\left[\begin{array}{cc}
1 & 0 \\
0 & T^{-T}
\end{array}\right]\right\|_{s}
\end{aligned}
$$

where $\Phi_{i}$ is a fixed complex matrix. The following lemma proven by Istepanian et al., (1998b) show that optimization problem (23) can be expressed by solving the other two associated optimization 
problems (24) and (26).

Lemma 4: Let

$$
v_{1}=\min _{\substack{x \in(0,+\infty) \\ y \in(-\infty,+\infty) \\ w \in(0,+\infty)}} f_{1}(x, y, w)
$$

where

$$
\begin{aligned}
& f_{1}(x, y, w) \\
& =\max _{i \in\{1, \cdots, m+2\}}\left\|\left[\begin{array}{ccc}
w & 0 & 0 \\
0 & x & 0 \\
0 & y & 1 / x
\end{array}\right] \Phi \Phi_{i}\left[\begin{array}{ccc}
1 / w & 0 & 0 \\
0 & 1 / x & 0 \\
0 & -y & x
\end{array}\right]\right\|_{s}
\end{aligned}
$$

Let

$$
v_{2}=\min _{\substack{x \in(-\infty,+\infty) \\ y \in(-\infty,+\infty) \\ z \in(0,+\infty) \\ w \in(0,+\infty)}} f_{2}(x, y, z, w)
$$

where

$$
\begin{aligned}
& f_{2}(x, y, z, w) \\
& =\max _{i \in\{1, \cdots, m+2}\left\|\left[\begin{array}{ccc}
w & 0 & 0 \\
0 & x & z \\
0 & \frac{x y-1}{z} & y
\end{array}\right] \Phi_{i}\left[\begin{array}{ccc}
\frac{1}{w} & 0 & 0 \\
0 & y & -z \\
0 & \frac{1-x y}{z} & x
\end{array}\right]\right\|_{s}
\end{aligned}
$$

Then $\quad v=\min \left(v_{1}, v_{2}\right)$. If $\quad v=v_{1} \quad$ and $\left(x_{o p t 1}, y_{o p t 1}, w_{o p t l}\right)$ is the optimal solution of problem (24), the optimal solution of problem (23) can be given as:

$$
T_{o p t}=\frac{1}{w_{o p t l}}-\left[\begin{array}{cc}
x_{o p t 1} & y_{o p t l} \\
0 & 1 / x_{o p t l}
\end{array}\right]
$$

If $U=U_{2}$ and $\left(x_{o p t 2}, y_{o p t 2}, z_{o p t 2}, w_{o p t 2}\right)$ is the optimal solution of problem (26), the optimal solution of problem (23) can be given as:

$$
T_{\text {opt }}=\frac{1}{w_{\text {opt } 2}}\left[\begin{array}{cc}
x_{\text {opt } 2} & \left(x_{o p t 2} y_{o p t 2}-1\right) / z_{o p t 2} \\
z_{\text {opt2 }} & y_{\text {opt } 2}
\end{array}\right]
$$

Evidently, the problems (24) and (26) are multivariable nonlinear programming problems which can be solved efficiently using the Rosenbrock algorithmin (Dixon, 1972).

\section{APPLICATION TO ELECTROHYDRAULIC ACTUATOR SYSTEM}

In order to illustrate how the optimization approach presented earlier can be used efficiently for the deltabased parameterization issues of optimal FWL SISO two order controller structures with improved stability bounds and minimum word-length requirements. We consider the following industrial electrohydraulic actuator PI control system to confirm our theoretical results.

The continuous time linearized model $P(s)$ of electrohydraulic actuator system is given by :

$$
\begin{aligned}
& A_{P}=\left[\begin{array}{cccc}
0 & 1 & 0 & 0 \\
-2.4667 \times 10^{5} & -62800 & 0 & 0 \\
-2.5485 \times 10^{22} & 0 & -551.13 & 0 \\
1.8595 \times 10^{2} & 0 & 0 & -29359
\end{array}\right], \\
& B_{P}=\left[\begin{array}{c}
0 \\
18933 \\
0 \\
0
\end{array}\right], C_{P}=\left[\begin{array}{llll}
0 & 0 & -2.2500 \times 10^{-4} & 4.0200 \times 10^{-4}
\end{array}\right]
\end{aligned}
$$

To provide a high bandwidth force control loop, a stabilized force feedback PI controller with a prefilter is designed:

$$
\frac{-1}{0.0001 s+1}\left(0.005+\frac{0.05}{s}\right)
$$

The sampling period chosen as $h=2^{-12}$. Using bilinear (Tustin) method we obtain the initial realization of $C(\delta)$ as:

$$
\begin{aligned}
& A_{C}^{0}=\left[\begin{array}{cc}
0 & 0 \\
1 & -4.5031 \times 10^{3}
\end{array}\right], B_{C}^{0}=\left[\begin{array}{l}
1 \\
0
\end{array}\right] \text {, } \\
& C_{C}^{0}=\left[\begin{array}{ll}
-10.179 & 45610
\end{array}\right] D_{C}^{0}=\left[-2.7518 \times 10^{3}\right]
\end{aligned}
$$

For problem (24). we set initial point $x_{01}=1, y_{01}=0, w_{01}=1$. The realization corresponding to $\left(x_{01}, y_{03}, w_{01}\right)$ is $X_{0}$. By using Rosenbrock iterative algorithm, we get the solution $x_{\text {opt } 1}=17.688, y_{\text {opt } 1}=253.86, w_{\text {opt } 1}=56.399 \quad$ and $v_{1}=5353.7158$. The new realization corresponding to $\left(x_{o p t 1}, y_{o p t 1}, w_{o p t 1}\right)$ is $X_{o p t 1}$. For problem (26). we set initial point $x_{02}=1, y_{02}=1, z_{02}=1, w_{02}=1$. The resultant realization corresponding to $\left(x_{02}, y_{02}, z_{02}, w_{02}\right)$ is $X_{2}$. By using Rosenbrock iterative algorithm, we get the solution $x_{o p t 2}=2.5207 \times 10^{2}, \quad y_{o p t 2}=2.3182 \times 10^{-3}$, $z_{\text {opt } 2}=5.6092 \times 10^{-2}, \quad w_{\text {opt } 2}=5.6776 \times 10$ and $v_{2}=5353.7027$. The rcalization corresponding to $\left(x_{o p t 2}, y_{o p t 2}, z_{o p t 2}, w_{o p t 2}\right)$ is given as:

$$
\begin{gathered}
X_{\text {opt } 2}=\left[\begin{array}{ccc}
-2.7518 \times 10^{-3} & -1.2974 \times 10^{-1} & 3.1908 \\
1.3162 \times 10^{-1} & -3.8316 & -132.27 \\
-3.1847 & -1.3034 \times 10^{2} & -4499.2
\end{array}\right] \\
\text { Hencc } v=\min \left(v_{1}, v_{2}\right)=v_{2}=5353.7027 \quad \text { and }
\end{gathered}
$$

$X_{\text {opr2 }}$ is the relevant optimal realization.

Table I Stability Measures and word lengths

\begin{tabular}{lll}
\hline Realization & $\mu_{1}$ & $\hat{B}_{s !}^{\min }$ \\
\hline$X_{0}$ & $1.6647 \times 10^{-5}$ & 31 \\
$X_{2}$ & $8.7076 \times 10^{-6}$ & 32 \\
$X_{o p t l}$ & $1.867862 \times 10^{-4}$ & 25 \\
$X_{0 p t 2}$ & $1.867866 \times 10^{-4}$ & 25 \\
\hline
\end{tabular}


Tablc 1 summarizes the results for the different finite realizations and show their relevant stability robustness measure $\mu_{1}$ and the stabilized word length $\hat{B}_{s 1}^{\min }$ for the electrohydraulic actuator sampled data system.

\section{CONCLUSION}

In this paper a new delta-based FWL stability measure is introduced. It has been shown that the relevant optimal FWL realizations for two order controller structures can be interpreted as a constrained nonlinear optimization problem. A numerical example using a PI electrohydraulic actuator sampled data control system is presented to verify the improved parametrization characteristics at high sampling rates. Ongoing work is currently underway to generalize the methodology to higher order controller structures with integrated robust formulations.

\section{ACKNOWLEDGEMENT}

The first and second authors would like to thank the Royal Society-London for their ex-agreement ChinaBritish research visit Grant (Ref: 652053. Q606/AJM) and their support to this work. The authors would like also to thank Dr. Ian Pratt from Department of Automatic Control and Systems Engincering, University of Sheffield, UK for providing the electrohydraulic actuator model parameters and the controller structures. The first author would like to thank the National Natural Science Foundation of China under Grant 69504010 and Cao Guangbiao Foundation of Zhejiang University.

\section{REFERENCES}

Chen, T. and B. A. Francis (199I). Input-output stability of sampled-data systems. IEEE Transactions on Automatic Contr., 36, pp. 50-58.

Dixon, L. C. W. (1972). Nonlinear Optimisation. The English Universitics Press Ltd, London.

Fialho, I. J. and T. T. Georgiou (1994). On stability and performance of sampled data systems subject to word length constraint. IEEE Trans. on Automatic Control, 39, pp. 2476-2481.

Gevers, M. and G. Li (1993). Parametrizations in Control, Estimation and Filtering Problems: Accuracy Aspects, Communications and Control Engineering Series. Springer Verlag, London.

Istepanian, R. H., I. Pratt, R. Goodall and S. Jones (1996). Effect of fixed point parameterization on the performance of active suspension control systems. Proc. of 13th IFAC World Congress, San Francisco, California, pp. 291-295.
Istepanian, R. H. (1997). Implementation issues for discrete PID algorithms using shift and delta operators parameterizations. Proceedings of 4th IFAC Workshop on Algorithms and Architectures for Real-Time Control. Vilamoura, Portugal, pp. 117-122.

Istepanian, R. H., G. Li, J. Wu and J. Chu (1998a). Analysis of sensitivity measures of finiteprecision digital controller structures with closcd-loop stability bounds. Proceedings IEE Control Theory and Application, 145, pp. 472478.

Istepanian, R. H., J. Wu, J. Chu and J. F. Whidborne (1998b). Maximizing lower bound stability measure of finite precision PID controller realization by nonlinear programming. Proceedings of American Control Conference, Philadelphia, USA, pp. 2596-2600.

Istepanian, R. H., J. Wu, J. F. Whidborne, J. Yan and S. E. Salcudean (1998c). FWL Stability issues of teleoperation motion-scaling control system. Proceedings of UKACC International Conference on Control'98, Swansea, UK, pp. 1676-1681.

Li, G. and M. Gevers (1993). Comparative study of finite wordlength effects in shift and delta operator parameterizations. IEEE Transactions on Automatic C'ontrol, 38, pp. 803-807.

Li, G. and M. Gevers (1996). On the structure of digital controllers in sampled data systems with FWL consideration. Proceedings of 35th Conference on Decision and Control, Kobe, Japan, pp. 919-920.

Madievski, A. G., B. D. O. Anderson and M. Gevers (1995). Optimum realizations of sampled-data controllers for FWL sensitivity minimization. Automatica, 31, pp. 367-379.

Middleton, R. H. and G. C. Goodwin (1990). Digital Control and Estimation: A Unified Approach. Prentice Hall, New Jersey.

Moroney, P., A. S. Willsky and P. K. Houpt (1980) The digital implementation of control compensators: The coefficient wordlength issue. IEEE Transactions on Automatic Control, 25, pp. 621-630.

Qiu, L., B. Bernhardsson, A. Rantzer, E. J. Davison, P. M. Young and J. C. Doyle (1993). On the real structured stability radius. Proceedings of 12th World IFAC Congress, Sydney, Australia, pp. $71-78$.

Roberts, R. A. and C. T. Mullis (1987), Digital Signal Processing. Addison-Wesley.

Whidborne, J. F., R. H. Istepanian, I. Njabeleke and J. Wu (1998). Optimal digital controller structure for a fluid power system subject to wordlengh constraints. Proceedings of UKACC International Conference on Control'98, Swansea, UK, pp. 146-151. 